\title{
Do we really understand how antibiotics work?
}

\author{
Michaela Wenzel*,1 iD \\ ${ }^{1}$ Department of Biology \& Biological Engineering, Division of Chemical Biology, Chalmers University of Technology, 41296 \\ Gothenburg, Sweden \\ *Author for correspondence: Tel.: +46 31772 2074; wenzelm@chalmers.se
}

\begin{abstract}
"Despite the pitfall of potentially increased toxicity, the rapid development of antimicrobial resistance has led to a re-evaluation of antibiotic drug candidates with structural and multiple targets that were previously regarded unspecific."
\end{abstract}

First draft submitted: 24 November 2019; Accepted for publication: 21 August 2020; Published online: 22 September 2020

Keywords: antibiotic resistance $\bullet$ antibiotics $\bullet$ in vivo $\bullet$ mechanism of action $\bullet$ mode of action analysis $\bullet$ multifunctional • multitarget

\section{The classical magic bullets}

The discovery of antibiotics, pioneered by personalities like Paul Ehrlich, Selman Waksman and Alexander Fleming, ushered in a new era of infection medicine. In the year 1900, Paul Ehrlich first described the concept of the 'magic bullet', a chemical that harms pathogens but not the host. The first successful 'magic bullet' was arsphenamine, which revolutionized the treatment of syphilis [1]. The serendipitous discovery of penicillin by Fleming in 1928 and the isolation of streptomycin and other antibiotics from soil bacteria by Waksman in the 1940s heralded the golden age of antibiotics. Since then, a plethora of antimicrobial substances have been identified and made available for medical use [2].

Penicillin impairs the synthesis of the bacterial cell wall and streptomycin inhibits ribosome function. Until today, these two processes are still the most common targets of clinically used antibiotics. However, there are many other known mechanisms of action of antibiotics and a variety of bacterial cell structures can be exploited as antibiotic targets. This includes, for example, the cell membrane, DNA, RNA or specific enzymes. Understanding the mechanisms underlying antibacterial activity is essential for efficient drug development and identifying its molecular target is a prerequisite for bringing a new drug to the market.

\section{A new era of drug development}

Today, the golden age of antibiotics has passed and the increasing resistance of bacteria to established antibiotic classes demands creative antibacterial solutions. Thus, drug candidates with not yet clinically exploited targets are favorable over derivatives of existing drugs. However, this approach no longer allows for straightforward target validation with established standard assays. Moreover, it has become abundantly clear that antibiotics with specific protein targets suffer from fast resistance development rates. For example, sulfonamides, targeting an enzyme involved in folate synthesis and rifampicin, targeting RNA polymerase, are famous for fast resistance development and are therefore applied in combination with other drugs [3,4]. In search of more durable solutions, antibiotic lead development has gradually shifted to antibiotic candidates with targets encoded by multiple genes, with structural or multiple targets [5].

Despite many successful antibiotic classes falling into this category [5], antibiotic drug development has had a strong focus on single-target antibiotic candidates for a considerable time. It has long been a common notion that only highly specific single-target drugs provide the required selectivity and therapeutic window to be used in patients with acceptable side effects. Multitarget drugs or compounds with structural targets, such as the cytoplasmic membrane, have long been regarded unspecific and likely to display off-target activity in humans. Indeed, some drugs with multiple and/or structural targets display serious side effects. Thus, polymyxins, which target both the

Future $\because$ Medicine 
outer and inner membrane of Gram-negative bacteria [6], may display severe nephrotoxicity and neurotoxicity [7]. However, other drugs with truly unspecific targets, such as nitrofurantoin, have been in clinical use for decades with manageable side effects and severe adverse reactions are rare [8]. Nitrofurantoin is a prodrug that upon activation by cellular reductases leads to the formation of reactive oxygen species, which damage cellular macromolecules such as DNA, proteins and lipids [8]. While both human and bacterial reductases activate nitrofurantoin, this process is much faster in bacteria, underlying its selectivity. Importantly, resistance against nitrofurantoin remains rare despite its decade-long use in treating urinary tract infections [8]. On the other hand, compounds with only one highly specific target in bacteria can still have off-target activity in humans and cause severe side effects or allergic reactions. Examples include the high incidence of severe nephrotoxicity and ototoxicity observed for many aminoglycosides and wide-spread allergies toward penicillins [9-11]. Moreover, several studies support the notion that the most effective antibiotics that we use in the clinic do, in fact, not target a single molecule but affect multiple processes in the bacterial cell [5,12].

These examples illustrate that the notion that single-target antibiotics are generally less toxic, must be abandoned. At the same time, it is clear that potential off-target activity of any antibiotic drug candidate needs to be carefully addressed. However, adverse effects observed in cell cultures or animal studies may vary significantly depending on the model used. For example, the antibiotic candidate rhodomyrtone is toxic to zebrafish when administered orally, but appears to be safe for oral consumption by humans [13]. Such host-dependent toxicity significantly complicates the preclinical assessment of antibiotic off-target activity.

Despite the pitfall of potentially increased toxicity, the rapid development of antimicrobial resistance has led to a re-evaluation of antibiotic drug candidates with structural and multiple targets that were previously regarded unspecific.

\section{Complex \& multiple mechanisms of action}

Some mechanisms of action presuppose multiple targets or at least multiple effects. For example, bacitracin binds to bactoprenol phosphate and affects both synthesis of cell wall peptidoglycan and cell wall teichoic acids, since both pathways share bactoprenol phosphate as a transmembrane carrier molecule for their respective precursors [14]. Membrane-active antibiotics, although possibly binding to only a single target, by default inhibit multiple cellular processes. Their interference with membrane functionality primarily impairs cell wall synthesis and cellular respiration, but also a multitude of other membrane-bound processes such as cell division, protein secretion, sporulation, or the synthesis of new membrane material [12]. Some antibiotics bind to two or more separate molecular targets. For example, polymyxins inhibit both the outer and inner membrane of Gram-negative bacteria [6] and the lantibiotic nisin inhibits cell wall biosynthesis by binding to lipid II and subsequently uses the undecaprenyl-coupled cell wall precursor as a docking molecule to form a transmembrane pore [15]. Nisin is also able to bind the intermediate lipid I adding another molecule to its target repertoire [16].

Interestingly, nisin also displays a two-staged mechanism of action: it selectively inhibits cell wall synthesis at low doses while pore formation requires a certain threshold concentration [17]. Similar concentration-dependent mode of action shifts have been observed for several antimicrobial compounds. For example, both the fatty acid synthesis inhibitor triclosan and the tetracycline-class ribosome inhibitor chelocardin additionally target the cytoplasmic membrane at higher concentrations [18-20]. Such dose-dependent dual mechanisms add another layer of complexity to the mechanistic diversity of antibiotic molecules.

While their advantages for resistance development are clear, the complex mechanisms of such drugs can be hard to fully elucidate. One prominent example is the lipopeptide antibiotic daptomycin, which, based on model membrane studies, was long believed to form pores in bacterial membranes [21]. This model was challenged, when it was discovered that daptomycin does not cause pores in bacteria but instead interferes with membrane domains that harbor the cell wall synthesis machinery [22]. A very recent study has now found that daptomycin is in fact a dual target antibiotic and binds both phosphatidylglycerol-containing membrane lipids and undecaprenyl-coupled cell wall precursors [23].

While the mechanisms of daptomycin has been elusive for a long time, surprising new activities are also being discovered for antibiotics that were assumed to be well characterized and already fully understood. For example, it was recently shown that the translation inhibitor tetracycline targets the bacterial cell membrane in addition to the ribosome and that this appears to be a common feature of the tetracycline class of antibiotics [20,24]. These examples illustrate how difficult it can be to fully understand antibiotic modes of action and it is well possible that other established antibiotics have different or secondary mechanisms that have so far escaped discovery. 


\section{Consequences for mode of action studies}

While compounds with multiple mechanisms have the promise to be superior to single-target drugs in terms of resistance development, they pose different challenges for the drug development process. Thus, such compounds are often poorly characterized and tools to appropriately analyze their complex mechanisms of action may be limited. Not only drug development, but also mode of action analysis has long been focused on single targets and target validation has often been performed in vitro, for example, with isolated enzymes, isolated cell wall precursors, or model membranes. Additionally, the decades-long focus of the pharmaceutical industry on single-target drugs fostered a mindset of settling for one mechanism of action, since additional targets were viewed as unspecific offtarget activity. Thus, one major difficulty has long been the limited availability of suitable assays to study complex antibiotic mechanisms in living bacterial cells. However, there is increasing evidence that mechanisms of action can differ substantially between in vitro and in vivo and that antibiotics may have additional mechanisms of action in living bacterial cells [12]. Thus, technologies to study antibiotic mechanisms in living bacteria are urgently needed to capture their true nature and complexity.

While the realization that successful antibiotics do have more than one single target has emerged rather slowly, the negative attitude toward multitarget antibiotics finally changed with the dramatic increase in drug-resistant bacteria. Modern drug development may now even aim at multiple mechanisms and no longer shies away from complex structural targets like the cell membrane. For example, the vancomycin derivatives telavancin and oritavancin carry a lipid tail, which allows them to interact with the cytoplasmic membrane in addition to binding lipid II [25].

With this newly found appreciation for complex and multiple targets the range of advanced in vivo techniques for mode of action analysis has increased as well. In particular, a range of cell biological tools to analyze complex structural targets like the cytoplasmic membrane or cell wall components have been developed and employed for antibiotic mode of action analysis with great success [22,26,27]. For example, the development of fluorescent amino acids to visualize active cell wall synthesis and the application of specialized lipid dyes to analyze membrane domain structure and fluidity now allow examining cellular processes that were not possible to observe in living bacteria before [22,27]. These developments now make it possible to analyze how antibiotics interact with bacterial cell structures and kill bacteria in impressive detail [13]. This cell biological toolbox is constantly expanding and new tools for fluorescence and high-resolution microscopy are being developed, hopefully allowing even more detailed insight into bacterial cell biology and antibiotic-killing mechanisms.

While it is a great advancement to microscopically observe bacterial cellular processes in real time, one perspective alone rarely elucidates the whole picture, especially not when it comes to something as complex as the interactions of antibiotic molecules with bacterial cells. Detailed in vivo approaches are the strongest, when combined with complementary techniques. Thus, the combination with systems biology approaches, such as genomics, proteomics or metabolomics, cutting-edge in vitro technologies, or molecular modeling have all been proven very successful in unraveling the details of complex antibiotic mechanisms [13,28,29].

Several novel fields are emerging in and around antibiotic research, from new strategies to discover antimicrobial compounds and new techniques to analyze them to approaches to resensitize bacteria to existing antibiotics and developing antibiotic alternatives, the recent surge in antimicrobial resistance research has inspired many exciting new approaches. For example, artificial intelligence and machine learning approaches have delivered the first antibiotic candidates [30-33] and the potential of the microbiome in fighting bacterial infections is just being discovered [34]. Likewise, novel inspiring tools for mode of action analysis are being developed, for example machine learning approaches, new cell biological tools, or new image analysis platforms [35-37].

Whatever exciting new discoveries the future of antimicrobial drug development holds, one thing has become abundantly clear, namely that different disciplines must join forces to truly understand all aspects of antibiotic action that are relevant for drug discovery. Only if we understand how antibiotics really work, will we be able to efficiently develop novel lead structures that have the potential to become more sustainable antibiotics than the single-target screening hits resulting from the genomics-driven approaches that have dominated the landscape of antibiotic hit discovery for the last decades.

\section{Financial \& competing interests disclosure}

The author has no relevant affiliations or financial involvement with any organization or entity with a financial interest in or financial conflict with the subject matter or materials discussed in the manuscript. This includes employment, consultancies, honoraria, stock ownership or options, expert testimony, grants or patents received or pending, or royalties.

No writing assistance was utilized in the production of this manuscript. 


\section{Open access}

This work is licensed under the Attribution-NonCommercial-NoDerivatives 4.0 Unported License. To view a copy of this license, visit http://creativecommons.org/licenses/by-nc-nd/4.0/

\section{References}

1. Cooper JE. A brief romance with magic bullets: René Dubos at the dawn of the antibiotic era. In: Silent Victories: The history and Practice ofPublic Health in Twentieth Century America. Ward JW, Warren C (Eds). Oxford University Press, NY, USA, 42-62 (2007).

2. Aminov RI. A brief history of the antibiotic era: lessons learned and challenges for the future. Front. Microbiol. 1, 134 (2010).

3. Sköld O. Sulfonamide resistance: mechanisms and trends. Drug Resist. Updat. 3(3), 155-160 (2000).

4. Chopra I. Bacterial RNA polymerase: a promising target for the discovery of new antimicrobial agents. Curr. Opin. Investig. Drugs 8(8), 600-607 (2007).

5. Brötz-Oesterhelt H, Brunner N. How many modes of action should an antibiotic have? Curr. Opin. Pharmacol. 8(5), 564-573 (2008).

6. Deris ZZ, Akter J, Sivanesan S et al. A secondary mode of action of polymyxins against Gram-negative bacteria involves the inhibition of NADH-quinone oxidoreductase activity. J. Antibiot. (Tokyo) 67(2), 147-151 (2014).

7. Wolinsky E, Hines JD. Neurotoxic and nephrotoxic effects of colistin in patients with renal disease. N. Engl. J. Med. 266, 759-762 (1962).

8. Squadrito FJ, del Portal D. In: Nitrofurantoin. StatPearls Publishing, FL, USA (2020).

9. Lopez-Novoa JM, Quiros Y, Vicente L, Morales AI, Lopez-Hernandez FJ. New insights into the mechanism of aminoglycoside nephrotoxicity: an integrative point of view. Kidney Int. 79(1), 33-45 (2011).

10. Petersen L, Rogers C. Aminoglycoside-induced hearing deficits - a review of cochlear ototoxicity. South African Fam. Pract. 57(2), 77-82 (2015).

11. Gonzalez-Estrada A, Radojicic C. Penicillin allergy: a practical guide for clinicians. Cleve. Clin. J. Med. 82(5), 295-300 (2015).

12. Gray DA, Wenzel M. Multitarget approaches against multiresistant superbugs. ACS Infect. Dis. 6(6), 1346-1365 (2020).

13. Saeloh D, Tipmanee V, Jim KK et al. The novel antibiotic rhodomyrtone traps membrane proteins in vesicles with increased fluidity. PLoS Pathog. 14(2), e1006876 (2018).

14. Weidenmaier C, Peschel A. Teichoic acids and related cell-wall glycopolymers in Gram-positive physiology and host interactions. Nat. Rev. Microbiol. 6(4), 276-287 (2008).

15. Brotz H, Josten M, Wiedemann I et al. Role of lipid-bound peptidoglycan precursors in the formation of pores by nisin, epidermin and other lantibiotics. Mol. Microbiol. 30(2), 317-327 (1998).

16. 't Hart P, Oppedijk SF, Breukink E, Martin NI. New insights into Nisin's antibacterial mechanism revealed by binding studies with synthetic lipid II analogues. Biochemistry 55(1), 232-237 (2016).

17. Wenzel M, Kohl B, Münch D et al. Proteomic response of Bacillus subtilis to lantibiotics reflects differences in interaction with the cytoplasmic membrane. Antimicrob. Agents Chemother. 56(11), 5749-5757 (2012).

18. Villalaín J, Mateo CR, Aranda FJ, Shapiro S, Micol V. Membranotropic effects of the antibacterial agent triclosan. Arch. Biochem. Biophys. 390(1), 128-136 (2001).

19. Escalada MG, Russella D, Maillard J-Y, Ochs D. Triclosan-bacteria interactions: single or multiple target sites? Lett. Appl. Microbiol. 41(6), 476-481 (2005).

20. Stepanek JJ, Lukezic T, Teichert I, Petkovic H, Bandow JE. Dual mechanism of action of the atypical tetracycline chelocardin. Biochim. Biophys. Acta 1864(6), 645-654 (2016).

21. Zhang T, Muraih JK, MacCormick B, Silverman J, Palmer M. Daptomycin forms cation- and size-selective pores in model membranes. Biochim. Biophys. Acta 1838(10), 2425-2430 (2014).

22. Müller A, Wenzel M, Strahl H et al. Daptomycin inhibits cell envelope synthesis by interfering with fluid membrane microdomains. Proc. Natl Acad. Sci. USA 113, E7077-E7086 (2016).

23. Grein F, Muller A, Scherer $\mathrm{KM}$ et al. $\mathrm{Ca}(2+)$-daptomycin targets cell wall biosynthesis by forming a tripartite complex with undecaprenyl-coupled intermediates and membrane lipids. Nat. Commun. 11(1), 1455 (2020).

24. Wenzel M, Dekker MP, Wang B et al. New flat embedding method for transmission electron microscopy reveals an unknown mechanism of tetracycline. bioRxiv doi:https://doi.org/doi:10.1101/820191 (2019) (Epub ahead of print).

25. Schneider T, Sahl HG. An oldie but a goodie - cell wall biosynthesis as antibiotic target pathway. Int. J. Med. Microbiol. 300(2-3), 161-169 (2010).

26. Nonejuie P, Burkart M, Pogliano K, Pogliano J. Bacterial cytological profiling rapidly identifies the cellular pathways targeted by antibacterial molecules. Proc. Natl Acad. Sci. USA 110(40), 16169-16174 (2013).

27. Kuru E, Velocity Hughes H, Brown PJ et al. In situ probing of newly synthesized peptidoglycan in live bacteria with fluorescent D-amino acids. Angew. Chem. Int. Ed. Engl. 51(50), 12519-12523 (2012). 
28. Scheinpflug K, Wenzel M, Krylova O et al. Antimicrobial peptide cWFW kills by combining lipid phase separation with autolysis. Sci. Rep. 7, 44332 (2017).

29. Omardien S, Drijfhout JW, van Veen $\mathrm{H}$ et al. Synthetic antimicrobial peptides delocalize membrane bound proteins thereby inducing a cell envelope stress response. Biochim. Biophys. Acta Biomembr. 1860(11), 2416-2427 (2018).

30. Cherkasov A, Hilpert $\mathrm{K}$, Jenssen $\mathrm{H}$ et al. Use of artificial intelligence in the design of small peptide antibiotics effective against a broad spectrum of highly antibiotic-resistant superbugs. ACS Chem. Biol. 4(1), 65-74 (2009).

31. Fjell CD, Jenssen $\mathrm{H}$, Hilpert $\mathrm{K}$ et al. Identification of novel antibacterial peptides by chemoinformatics and machine learning. J. Med. Chem. 52(7), 2006-2015 (2009).

32. Mikut R, Ruden S, Reischl M, Breitling F, Volkmer R, Hilpert K. Improving short antimicrobial peptides despite elusive rules for activity. Biochim. Biophys. Acta 1858(5), 1024-1033 (2016).

33. Stokes JM, Yang K, Swanson K et al. A deep learning approach to antibiotic discovery. Cell 180(4), 688-702.e13 (2020).

34. Relman DA, Lipsitch M. Microbiome as a tool and a target in the effort to address antimicrobial resistance. Proc. Natl Acad. Sci. USA 115(51), 12902-12910 (2018).

35. Peach KC, Bray WM, Winslow D, Linington PF, Linington RG. Mechanism of action-based classification of antibiotics using high-content bacterial image analysis. Mol. Biosyst. 9(7), 1837-1848 (2013).

36. Stone MRL, Butler MS, Phetsang W, Cooper MA, Blaskovich MAT. Fluorescent antibiotics: new research tools to fight antibiotic resistance. Trends Biotechnol. 36(5), 523-536 (2018).

37. Zoffmann S, Vercruysse M, Benmansour F et al. Machine learning-powered antibiotics phenotypic drug discovery. Sci. Rep. 9(1), 5013 (2019). 
